\title{
Spectrum perturbations of compact operators in a Banach space
}

https://doi.org/10.1515/math-2019-0085

Received September 18, 2018; accepted August 12, 2019

Abstract: For an integer $p \geq 1$, let $\Gamma_{p}$ be an approximative quasi-normed ideal of compact operators in a Banach space with a quasi-norm $N_{\Gamma_{p}}($.$) and the property$

$$
\sum_{k=1}^{\infty}\left|\lambda_{k}(A)\right|^{p} \leq a_{p} N_{\Gamma_{p}}^{p}(A) \quad\left(A \in \Gamma_{p}\right)
$$

where $\lambda_{k}(A)(k=1,2, \ldots)$ are the eigenvalues of $A$ and $a_{p}$ is a constant independent of $A$. Let $A, \tilde{A} \in \Gamma_{p}$ and

$$
\Delta_{p}(A, \tilde{A}):=N_{\Gamma_{p}}(A-\tilde{A}) \exp \left[a_{p} b_{p}^{p}\left(1+\frac{1}{2}\left(N_{\Gamma_{p}}(A+\tilde{A})+N_{\Gamma_{p}}(A-\tilde{A})\right)\right)^{p}\right],
$$

where $b_{p}$ is the quasi-triangle constant in $\Gamma_{p}$. It is proved the following result: let $I$ be the unit operator, $I-A^{p}$ be boundedly invertible and

$$
\Delta_{p}(A, \tilde{A}) \exp \left[\frac{a_{p} N_{\Gamma_{p}}^{p}(A)}{\psi_{p}(A)}\right]<1,
$$

where $\psi_{p}(A)=\inf _{k=1,2, \ldots}\left|1-\lambda_{k}^{p}(A)\right|$. Then $I-\tilde{A}^{p}$ is also boundedly invertible. Applications of that result to the spectrum perturbations of absolutely $p$-summing and absolutely $(p, 2)$ summing operators are also discussed. As examples we consider the Hille-Tamarkin integral operators and matrices.

Keywords: Banach space, compact operators, perturbations, absolutely $p$-summing operators, absolutely $(p, 2)$-summing operators, integral operators, infinite matrices

MSC: 47A10, 47A55, 47B10, 47A75

\section{Introduction and statement of the main result}

Roughly speaking, the spectrum perturbation theory for linear operators consists of two approaches. In the framework of the first one some structure on the error is imposed; for example, they may be analytic functions of a complex variable. The problem is then to determine how this structure affects the perturbed spectrum: e.g., when are they analytic functions of the variable, what kind of paths do they follow in the complex plane? That approach is well developed. For various results of this kind see for instance the book by Kato [1]. In the framework of the second approach the errors are unstructured and perturbations are bounded in terms of some norm of the errors. That approach in the case of operators in a Banach space to the best of our knowledge is at an early stage of development. Below we suggest perturbation results for compact operators in a Banach space, which are connected with the second approach.

Throughout this paper $X$ is a Banach space with the approximation property [2], the unit operator $I$ and norm $\|\cdot\|_{X}=\|\cdot\|,(\mathcal{B}(X))$ is the algebra of all bounded linear operators in $X$. For a compact operator $A, \lambda_{k}(A)$

`Corresponding Author: Michael Gil': Department of Mathematics, Ben Gurion University of the Negev, P.0. Box 653, BeerSheva 84105, Israel; E-mail: gilmi@bezeqint.net 
$(k=1,2, \ldots)$ are the eigenvalues counted with their algebraic multiplicities. A point $\lambda \in \mathbb{C}$ is said to be $\Phi$ regular for $A$ if $I-\lambda A$ is boundedly invertible; $\sigma_{\Phi}(A)$ denotes the Fredholm spectrum (the complement of all $\Phi$-regular points in the closed complex plane).

For an integer $p \geq 1$ introduce the two-sided quasi-normed ideal $\Gamma_{p}$ of compact operators in $(\mathcal{B}(X))$ with a quasi-norm $N_{\Gamma_{p}}($.$) and the property$

$$
\sum_{k=1}^{\infty}\left|\lambda_{k}(A)\right|^{p} \leq a_{p} N_{\Gamma_{p}}^{p}(A) \quad\left(A \in \Gamma_{p}\right),
$$

where $a_{p}$ is a constant independent of $A$, and $\Gamma_{p}$ is approximative (i.e. the set of all finite rank operators is dense in $\Gamma_{p}$ ). Below $b_{p}$ denotes the quasi-triangle constant in $\Gamma_{p}$ :

$$
N_{\Gamma_{p}}(A+\tilde{A}) \leq b_{p}\left(N_{\Gamma_{p}}(A)+N_{\Gamma_{p}}(\tilde{A})\right)\left(A, \tilde{A} \in \Gamma_{p}\right) .
$$

For the theory of the approximative normed and quasi-normed ideals see [2,3] and references given therein. In the sequel constant $a_{p}$ in (1.1) will be called the eigenvalue constant.

Put

$$
\Delta_{p}(A, \tilde{A}):=N_{\Gamma_{p}}(A-\tilde{A}) \exp \left[a_{p} b_{p}^{p}\left(1+\frac{1}{2}\left(N_{\Gamma_{p}}(A+\tilde{A})+N_{\Gamma_{p}}(A-\tilde{A})\right)\right)^{p}\right]
$$

and

$$
\psi_{p}(A)=\inf _{k=1,2, \ldots}\left|1-\lambda_{k}^{p}(A)\right| .
$$

Now we are in a position to formulate the main result of the paper.

Theorem 1.1. For an integer $p \geq 1$, let $A, \tilde{A} \in \Gamma_{p}$ and $I-A^{p}$ be boundedly invertible. If, in addition,

$$
\Delta_{p}(A, \tilde{A}) \exp \left[\frac{a_{p} N_{\Gamma_{p}}^{p}(A)}{\psi_{p}(A)}\right]<1,
$$

then $I-\tilde{A}^{p}$ is also boundedly invertible.

The proof of this theorem is presented in the next section. Replacing in Theorem $1.1 A$ and $\tilde{A}$ by $\lambda A$ and $\lambda \tilde{A}$, respectively, we get the following result.

Corollary 1.2. Let $A, \tilde{A} \in \Gamma_{p}$ and $\lambda^{p} \notin \sigma_{\Phi}\left(A^{p}\right)$. If, in addition,

$$
\Delta_{p}(\lambda A, \lambda \tilde{A}) \exp \left[\frac{a_{p} N_{\Gamma_{p}}^{p}(\lambda A)}{\psi_{p}(\lambda A)}\right]<1
$$

then $\lambda^{p}$ is $\Phi$-regular also for $\tilde{A}^{p}$.

From this corollary it follows

Corollary 1.3. Let $A, \tilde{A} \in \Gamma_{p}$ and $\mu^{p} \in \sigma_{\Phi}\left(\tilde{A}^{p}\right)$. Then either $\mu^{p} \in \sigma_{\Phi}\left(A^{p}\right)$, or

$$
\Delta_{p}(\mu A, \mu \tilde{A}) \exp \left[\frac{a_{p} N_{\Gamma_{p}}^{p}(\mu A)}{\psi_{p}(\mu A)}\right] \geq 1 .
$$

Note that (1.3) can be rewritten as

$$
|\mu| N_{\Gamma_{p}}(A-\tilde{A}) \exp \left[\frac{a_{p}|\mu|^{p} N_{\Gamma_{p}}^{p}(A)}{\psi_{p}(\mu A)}+a_{p} b_{p}^{p}\left(1+\frac{|\mu|}{2}\left(N_{\Gamma_{p}}(A+\tilde{A})+N_{\Gamma_{p}}(A-\tilde{A})\right)\right)^{p}\right] \geq 1 .
$$




\section{Proof of Theorem 1.1}

For an $A \in \Gamma_{1}$ introduce the determinant by

$$
\operatorname{det}(I-A)=\prod_{k=1}^{\infty}\left(1-\lambda_{k}(A)\right) .
$$

Obviously,

$$
|\operatorname{det}(I-A)| \leq \prod_{k=1}^{\infty}\left(1+\left|\lambda_{k}(A)\right|\right) \leq \exp \left[\sum_{k=0}^{\infty}\left|\lambda_{k}(A)\right|\right] .
$$

So from (1.1) we have

$$
|\operatorname{det}(I-A)| \leq \exp \left[a_{1} N_{\Gamma_{1}}(A)\right] .
$$

Hence, the convergence of the product follows. Since $\Gamma_{1}$ is approximative, we get

$$
\lim _{n \rightarrow \infty} \operatorname{det}\left(I-A_{n}\right)=\operatorname{det}(I-A)
$$

for a sequence $\left\{A_{n}\right\}$ of $n$-dimensional operators $(n<\infty)$ converging to $A$ in $N_{\Gamma_{1}}($.).

Various approaches to the determinants of operators in a Banach space can be found, in particular, in the well-known publications $[2,4,5]$.

Similarly, if $A \in \Gamma_{p}$ for $p>1$, we can write

$$
\operatorname{det}\left(I-A^{p}\right)=\prod_{k=1}^{\infty}\left(1-\lambda_{k}^{p}(A)\right)
$$

and according to (1.1)

$$
\left|\operatorname{det}\left(I-A^{p}\right)\right| \leq \exp \left[a_{p} N_{\Gamma_{p}}^{p}(A)\right]
$$

Lemma 2.1. Let $A, \tilde{A} \in \Gamma_{p}$. Then

$$
\left|\operatorname{det}\left(I-A^{p}\right)-\operatorname{det}\left(I-\tilde{A}^{p}\right)\right| \leq \Delta_{p}(A, \tilde{A}) .
$$

Proof. Let $A$ and $\tilde{A}$ be $n$-dimensional $(n<\infty)$. Consider the function

$$
f(\lambda)=\operatorname{det}\left(I-\left[\frac{1}{2}(A+\tilde{A})+\lambda(A-\tilde{A})\right]\right) .
$$

First assume that $I-\frac{1}{2}(A+\tilde{A})$ is invertible. Then

$$
\begin{aligned}
f(\lambda) & \left.=\operatorname{det}\left[I-\frac{1}{2}(A+\tilde{A})\right)\left(I-\lambda\left(I-\frac{1}{2}(A+\tilde{A})\right)^{-1}(A-\tilde{A})\right)\right] \\
& =\operatorname{det}\left(I-\frac{1}{2}(A+\tilde{A})\right) \operatorname{det}(I-\lambda C),
\end{aligned}
$$

where $C=\left(I-\frac{1}{2}(A+\tilde{A})\right)^{-1}(A-\tilde{A})$. But

$$
\operatorname{det}(I-\lambda C)=\prod_{k=1}^{n}\left(1-\lambda \lambda_{k}(C)\right)
$$

is a polynomial. So $f(\lambda)$ is a polynomial. Similarly, we can prove that

$$
\operatorname{det}\left(I-\left[\frac{1}{2}\left(A^{p}+\tilde{A}^{p}\right)+\lambda\left(A^{p}-\tilde{A}^{p}\right)\right]\right)
$$

is a polynomial, if $I-\frac{1}{2}\left(A^{p}+\tilde{A}^{p}\right)$ is invertible. Making use of Lemma 1.4.1 [6] (see also [7]), according to (2.1) we get (2.2). If $I-\frac{1}{2}\left(A^{p}+\tilde{A}^{p}\right)$ is not invertible, then (2.2) can be proved by a small perturbation of the considered operators and continuity of determinants. So for finite dimensional operators the lemma is proved. The approximativity of $\Gamma_{p}$ implies the required result. 
Corollary 2.2. Let $A, \tilde{A} \in \Gamma_{p}$ and

$$
\left|\operatorname{det}\left(I-A^{p}\right)\right|>\Delta_{p}(A, \tilde{A}) .
$$

Then

$$
\left|\operatorname{det}\left(I-\tilde{A}^{p}\right)\right| \geq\left|\operatorname{det}\left(I-A^{p}\right)\right|-\Delta_{p}(A, \tilde{A})>0 .
$$

Lemma 2.3. If the condition

$$
\sum_{k=1}^{\infty}\left|\lambda_{k}(A)\right|<\infty
$$

holds and $1 \notin \sigma_{\Phi}(A)$, then

$$
|\operatorname{det}(I-A)| \geq \exp \left[-\frac{1}{\psi_{1}(A)} \sum_{k=1}^{\infty}\left|\lambda_{k}(A)\right|\right] .
$$

Proof. By the usual procedure for the calculations of an extremum we find that $\max _{x \geq 0} e^{-x} x=1 / e$. Hence

$$
\frac{1}{|1-z|} e^{-\frac{1}{|1-z|}} \leq 1 / e \quad(z \in \mathbb{C})
$$

and

$$
|1-z| \geq e^{1-\frac{1}{|1-z|}}=e^{(|1-z|-1) /|1-z|} \geq e^{(1-|z|-1) /|1-z|}=e^{-\frac{|z|}{|1-z|}} .
$$

So if $1 \notin \sigma_{\Phi}(A)$, then

Hence

$$
\left|1-\lambda_{k}(A)\right| \geq \exp \left[-\frac{\left|\lambda_{k}(A)\right|}{\left|1-\lambda_{k}(A)\right|}\right] \geq \exp \left[-\frac{\left|\lambda_{k}(A)\right|}{\psi_{1}(A)}\right]
$$

$$
|\operatorname{det}(I-A)|=\prod_{k=1}^{\infty}\left|1-\lambda_{k}(A)\right| \geq \exp \left[-\frac{1}{\psi_{1}(A)} \sum_{k=1}^{\infty}\left|\lambda_{k}(A)\right|\right]
$$

as claimed.

Now let $A \in \Gamma_{p}$. From the previous lemma, due to (1.1)

$$
\left|\operatorname{det}\left(I-A^{p}\right)\right| \geq \exp \left[-\frac{a_{p} N_{\Gamma_{p}}^{p}(A)}{\psi_{p}(A)}\right] \quad\left(1 \notin \sigma_{\Phi}\left(A^{p}\right)\right) .
$$

Corollary 2.2 implies

Corollary 2.4. Let $A, \tilde{A} \in \Gamma_{p}$ for an integer $p \geq 1$. If, $1 \notin \sigma_{\Phi}\left(A^{p}\right)$ and

$$
\exp \left[-\frac{a_{p} N_{\Gamma_{p}}^{p}(A)}{\psi_{p}(A)}\right]>\Delta_{p}(A, \tilde{A}),
$$

then

$$
\left|\operatorname{det}\left(I-\tilde{A}^{p}\right)\right| \geq \exp \left[-\frac{a_{p}^{p} N_{\Gamma_{p}}^{p}(A)}{\psi_{p}(A)}\right]-\Delta_{p}(A, \tilde{A})>0 .
$$

The assertion of Theorem 1.1 directly follows from Corollary 2.4.

\section{Particular cases}

\subsection{Absolutely $p$-summing operators}

An operator $A \in(\mathcal{B}(X))$ is said to be absolutely $p$-summing $(1 \leq p<\infty)$, if there is a constant $v$, such that regardless of a natural number $m$ and regardless of the choice $x_{1}, \ldots, x_{m} \in X$ we have

$$
\left[\sum_{k=1}^{m}\left\|A x_{k}\right\|^{p}\right]^{1 / p} \leq v \sup \left\{\left[\sum_{k=1}^{m}\left|\left\langle x^{\star}, x_{k}\right\rangle\right|^{p}\right]^{1 / p}: x^{\star} \in X^{\star},\left\|x^{*}\right\|=1\right\} .
$$


Here $\langle.,$.$\rangle means the functional on X, X^{*}$ means the space adjoint to $X[2,3,8,9]$. The least $v$ for which this inequality holds is a norm and is denoted by $\pi_{p}(A)$. The set of absolutely $p$-summing operators in $X$ with the finite norm $\pi_{p}$ is a normed ideal in the set of bounded linear operators, which is denoted by $\Pi_{p}$, cf. [2].

As is well-known,

$$
\sum_{k=1}^{\infty}\left|\lambda_{k}(A)\right|^{p} \leq \pi_{p}^{p}(A) \quad\left(A \in \Pi_{p} ; 2 \leq p<\infty\right),
$$

cf. Theorem 17.4.3 from [9] (see also Theorem 3.7.2 from [2, p. 159]). Thus, $\Pi_{p}(p \geq 2)$ has the properties of ideal $\Gamma_{p}$. Besides, $N_{\Gamma_{p}}(A)=\pi_{p}(A), b_{p}=1$ and $a_{p}=1$.

\subsection{Ideal $\varepsilon_{p}$ and absolutely $(p, 2)$-summing operators}

Recall [2, p. 79] that $s_{n}(T)(n=1.2, \ldots)$ is called the $n$-th $s$-number ( $n$-th singular number) of $T \in(\mathcal{B}(X))$, if the following conditions are satisfied:

$$
\begin{aligned}
& \left(S_{1}\right)\|T\|=s_{1}(T) \geq s_{2}(T) \geq \ldots \geq 0 ; \\
& \left(S_{2}\right) s_{n+m-1}(S+T) \leq s_{m}(T)+s_{n}(S) \quad(S \in(\mathcal{B}(X))) ; \\
& \left(S_{3}\right) s_{n}\left(A_{1} T A_{2}\right) \leq\left\|A_{1}\right\| s_{n}(T)\left\|A_{2}\right\|\left(A_{1}, A_{2} \in(\mathcal{B}(X))\right) ; \\
& \left(S_{4}\right) \quad \text { If } \operatorname{rank}(T)<n, \text { then } s_{n}(T)=0 ; \\
& \left(S_{5}\right) s_{n}\left(I_{l_{n}^{2}}\right)=1 .
\end{aligned}
$$

Here $I_{l_{n}^{2}}$ is the unit operator in the $n$-dimensional Hilbert space $l_{n}^{2}$ with the traditional scalar product.

Let $L\left(l^{2}, X\right)$ denote the space of linear operators acting from the Hilbert space $l^{2}$ with the traditional scalar product into $X$. The $n$-th Weyl number of $T \in(\mathcal{B}(X)))$ is defined by

$$
x_{n}(T):=\sup \left\{a_{n}(T Z): Z \in L\left(l^{2}, X\right),\|Z\|=1\right\},
$$

where $a_{n}(T)$ is the $n$-th approximation number defined by

$$
a_{n}(T):=\inf \left\{\left\|T-T_{n}\right\|: T_{n} \in(\mathcal{B}(X)), \operatorname{rank} T_{n}<n\right\} .
$$

$x_{n}(T)$ is an s-number with the sub-multiplicative property

$$
\left(S_{6}\right) x_{n+m-1}(T S) \leq x_{n}(T) x_{m}(S) \quad(S, T \in(\mathcal{B}(X))),
$$

cf. [2, Theorem 2.4.14] and [2, Proposition 2.4.17]. For an integer $p \geq 1$, let $\varepsilon_{p}$. be the set of compact operators $A$ acting in $X$ and satisfying

$$
N_{\mathcal{E}_{p}}(A):=\left(\sum_{k=1}^{\infty} x_{k}^{p}(A)\right)^{1 / p}<\infty .
$$

Since $x_{k}(A) \leq x_{k-1}(A)$ and $x_{2 k-1}(A+\tilde{A}) \leq x_{k}(A)+x_{k}(\tilde{A})$, we have

$$
\sum_{k=1}^{\infty} x_{k}^{p}(A+\tilde{A})=\sum_{j=1}^{\infty} x_{2 j-1}^{p}(A+\tilde{A})+x_{2 j}^{p}(A+\tilde{A}) \leq 2 \sum_{j=1}^{\infty} x_{2 j-1}^{p}(A+\tilde{A}) \leq 2 \sum_{j=1}^{\infty}\left(x_{j}(A)+x_{j}(\tilde{A})\right)^{p} .
$$

By the Minkovsky inequality

$$
\left(\sum_{j=1}^{\infty}\left(x_{j}(A)+x_{j}(\tilde{A})\right)^{p}\right)^{1 / p} \leq\left(\sum_{j=1}^{\infty} x_{j}^{p}(A)\right)^{1 / p}+\left(\sum_{j=1}^{\infty} x_{j}^{p}(\tilde{A})\right)^{1 / p} .
$$

Then

$$
N_{\varepsilon_{p}}(A+\tilde{A}) \leq 2^{1 / p}\left(N_{\varepsilon_{p}}(A)+N_{\varepsilon_{p}}(\tilde{A})\right)
$$


So $\varepsilon_{p}$ is a quasinormed ideal with the quasi-triangular constant $b_{p}=2^{1 / p}$. It is approximative, cf. [2, 3]. We need the following Weyl type inequality:

$$
\sum_{k=1}^{\infty}\left|\lambda_{k}(A)\right|^{p} \leq c_{p}^{p} \sum_{k=1}^{\infty} x_{k}^{p}(A)=c_{p}^{p} N_{\mathcal{E}_{p}}^{p}(A)
$$

with

$$
c_{p}=2^{1 / p} \sqrt{2 e} .
$$

cf. [3, Theorem 2.a.6, p. 85].

So $\varepsilon_{p}$ is an example of ideal $\Gamma_{p}$ with $N_{\Gamma_{p}}(A)=N_{\mathcal{E}_{p}}(A), a_{p}=c_{p}^{p}$ and $b_{p}=2^{1 / p}$.

About the recent investigations of the singular numbers and Weyl type inequalities see [10]-[16].

Let us point an estimate for $N_{\varepsilon_{p}}(A)$. To this end recall that an $A \in(\mathcal{B}(X))$ is said to be absolutely $(p, q)$ summing $(p \geq q)$, if there is a constant $v$ such that regardless a natural number $m$ and regardless of the choice $x_{1}, \ldots, x_{m} \in X$ we have

$$
\left[\sum_{k=1}^{m}\left\|A x_{k}\right\|^{p}\right]^{1 / p} \leq v \sup \left\{\left[\sum_{k=1}^{m}\left|\left\langle x^{*}, x_{k}\right\rangle\right|^{q}\right]^{1 / q}: x^{*} \in X^{*},\left\|x^{*}\right\|=1\right\}
$$

cf. $[2,3,8,9]$. The least $v$ for which this inequality holds is denoted by $\pi_{p, q}(A)$. The set of absolutely $(p, q)$ summing operators is denoted by $\Pi_{p, q}$.

Due to [9, Theorem 16.3.1] $\pi_{p, q}$ is a norm and $\Pi_{p, q}$ with that norm is a Banach space. If $A \in \Pi_{p, q}$, then $\|A\| \leq \pi_{p, q}(A)$, since

$$
\|A x\|=\left[\|A x\|^{p}\right]^{1 / p} \leq \pi_{p, q}(A) \sup \left\{\left[\left|\left\langle x^{\star}, x\right\rangle\right|^{q}\right]^{1 / q}: x^{\star} \in X^{\star},\left\|x^{\star}\right\|=1\right\} \leq \pi_{p, q}(A)\|x\|
$$

for any $x \in X$. If, in addition $R$ and $S$ are bounded operators acting in $X$, then $\pi_{p, q}(S A R) \leq\|R\|_{X}\|S\|_{x} \pi_{p, q}(A)$.

We need Corollary 2.a.3 from [3, p. 81] (see also Corollary 17.2.2 from [9, p. 293]), which asserts the following: if $A \in \Pi_{p_{0}, 2}\left(2 \leq p_{0}<\infty\right)$, then

$$
x_{n}(A) \leq \frac{\pi_{p_{0}, 2}(A)}{n^{1 / p_{0}}}(n=1,2, \ldots) .
$$

Hence, for any $p>p_{0}$ we have

$$
N_{\varepsilon_{p}}(A)=\left(\sum_{k=1}^{\infty} x_{n}^{p}(A)\right)^{1 / p} \leq \pi_{p_{0}, 2}(A)\left(\sum_{k=1}^{\infty} \frac{1}{k^{p / p_{0}}}\right)^{1 / p}=\zeta^{1 / p}\left(p / p_{0}\right) \pi_{p_{0}, 2}(A) \quad\left(A \in \Pi_{p_{0}, 2}\right),
$$

where

$$
\zeta(z)=\sum_{k=1}^{\infty} \frac{1}{k^{z}}(\Re z>1)
$$

is the Riemann zeta-function.

\section{Additional upper bounds for determinants}

Lemma 4.1. For an integer $p \geq 1$ and $A \in \Gamma_{p}$ one has

$$
\left|\operatorname{det}\left(I-A^{p}\right)\right| \leq \psi_{p}(A) \exp \left[a_{p} N_{\Gamma p}^{p}(A)\right] .
$$

Proof. Evidently,

$$
\left|\operatorname{det}\left(I-A^{p}\right)\right|=\left|1-\lambda_{m}^{p}(A)\right| \prod_{k=1, k \neq m}^{\infty}\left|1-\lambda_{k}^{p}(A)\right| \leq\left|1-\lambda_{m}^{p}(A)\right| \exp \left[\sum_{k=1}^{\infty}\left|\lambda_{k}(A)\right|^{p}\right]
$$


for any $m \geq 1$. Taking into account (1.1) and choosing $m$ in such a way that $\left|1-\lambda_{m}^{p}(A)\right|=\psi_{p}(A)$, we prove the lemma.

Furthermore, let $E_{p}(z)$ be the Weierstrass primary factor:

$$
E_{1}(z)=(1-z) ; E_{p}(z)=(1-z) \exp \left[\sum_{m=1}^{p-1} \frac{z^{m}}{m}\right] \quad(p=2,3, \ldots ; z \in \mathbb{C}) .
$$

Put

$$
\gamma_{p}:=\frac{p-1}{p}(p \neq 1 ; p \neq 3) \text { and } \gamma_{1}=\gamma_{3}=1
$$

According to Theorem 1.5.3 [6],

$$
\left|E_{p}(z)\right| \leq \exp \left[\gamma_{p}|z|^{p}\right](z \in \mathbb{C}) .
$$

For an $A \in \Gamma_{p}, p \geq 2$, introduce the $p$-regularized determinant by

$$
\operatorname{det}_{p}(I-A):=\prod_{k=1}^{\infty} E_{p}\left(\lambda_{k}(A)\right)
$$

Due to (1.1) and (4.1)

$$
\left|\operatorname{det}_{p}(I-A)\right| \leq \exp \left[\gamma_{p} \sum_{k=1}^{\infty}\left|\lambda_{k}(A)\right|^{p}\right] \leq \exp \left[a_{0} \gamma_{p} N_{\Gamma p}^{p}(A)\right](p \geq 2),
$$

and therefore the product converges.

Lemma 4.2. For an integer $p \geq 2$ and any $A \in \Gamma_{p}$ one has

$$
\left|\operatorname{det}_{p}(I-A)\right| \leq \psi_{1}(A) \exp \left[\sum_{k=1}^{p-1} \frac{r_{S}^{k}(A)}{k}\right] \exp \left[a_{p} \gamma_{p} N_{\Gamma p}^{p}(A)\right],
$$

where $r_{s}(A)$ is the spectral radius of $A$.

Proof. By (4.1) and (1.1),

$$
\begin{aligned}
\left|\operatorname{det}_{p}(I-A)\right| & =\left|E\left(\lambda_{m}(A)\right)\right| \prod_{k=1, k \neq m}^{\infty}\left|E\left(\lambda_{k}(A)\right)\right| \leq\left|E\left(\lambda_{m}(A)\right)\right| \exp \left[\gamma_{p} \sum_{k=1, k \neq m}^{\infty}\left|\lambda_{k}(A)\right|^{p}\right] \\
& \leq\left|E\left(\lambda_{m}(A)\right)\right| \exp \left[a_{p} \gamma_{p} N_{\Gamma p}^{p}(A)\right]
\end{aligned}
$$

for any $m \geq 1$. But

$$
\left|E_{p}\left(\lambda_{m}(A)\right)\right|=\left|1-\lambda_{m}(A)\right|\left|\exp \left[\sum_{k=1}^{p-1} \frac{\lambda_{m}(A)^{k}}{k}\right]\right| \leq\left|1-\lambda_{m}(A)\right| \exp \left[\sum_{k=1}^{p-1} \frac{r_{S}^{k}(A)}{k}\right] .
$$

So

$$
\left|\operatorname{det}_{p}(I-A)\right| \leq\left|1-\lambda_{m}(A)\right| \exp \left[\sum_{k=1}^{p-1} \frac{r_{S}^{k}(A)}{k}\right] \exp \left[a_{p} \gamma_{p} N_{\Gamma p}^{p}(A)\right] .
$$

Hence, choosing $m$ in such a way that $\left|1-\lambda_{m}(A)\right|=\psi_{1}(A)$, we prove the lemma. 


\section{Hille-Tamarkin integral operators "close" to Volterra ones}

In this section and in the next one, we consider some concrete integral and matrix operators. We need the following result.

Corollary 5.1. Let $W \in \Gamma_{p}$ be a quasi-nilpotent operator (i.e. its spectrum is $\{0\}$ ). Then for an arbitrary $\tilde{A} \in \Gamma_{p}$ one has

$$
\left|\operatorname{det}\left(I-\tilde{A}^{p}\right)-1\right| \leq \Delta_{p}(W, \tilde{A}) .
$$

Indeed, this result is due to Lemma 2.1 and the equality $\operatorname{det}\left(I-W^{p}\right)=1$.

Let $L^{p}=L^{p}(0,1)(2 \leq p<\infty)$ be the space of scalar functions $f$ defined on $[0,1]$ and endowed the norm

$$
\|f\|=\left[\int_{0}^{1}|f(t)|^{p} d t\right]^{1 / p} .
$$

Let $K: L^{p} \rightarrow L^{p}$ be the operator defined by

$$
(K f)(t)=\int_{0}^{1} k(t, s) f(s) d s \quad\left(f \in L^{p}, 0 \leq t \leq 1\right),
$$

whose kernel $k$ defined on $[0,1]^{2}$ satisfies the condition

$$
\hat{k}_{p}(K):=\left[\int_{0}^{1}\left(\int_{0}^{1}|k(t, s)|^{p^{\prime}} d s\right)^{p / p^{\prime}} d t\right]^{1 / p}<\infty,
$$

where $1 / p+1 / p^{\prime}=1$. Then $K$ is called a $\left(p, p^{\prime}\right)$-Hille-Tamarkin integral operator.

As is well known, [8, p. 43], any $\left(p, p^{\prime}\right)$-Hille-Tamarkin operator $K$ is an absolutely $p$-summing operator with $\pi_{p}(K) \leq \hat{k}_{p}(K)$. Let the operator $V$ be defined by

$$
(V f)(t)=\int_{0}^{t} k(t, s) f(s) d s \quad\left(f \in L^{p}\right) .
$$

This operator is quasi-nilpotent. With $\Gamma_{p}=\Pi_{p}$ we have

$$
\Delta_{p}(K, V)=\pi_{p}(K-V) \exp \left[\left(1+\frac{1}{2}\left(\pi_{p}(K+V)+\pi_{p}(K-V)\right)\right)^{p}\right] \leq \hat{\Delta}_{p}(K, V),
$$

where

$$
\hat{\Delta}_{p}(K, V):=\hat{k}_{p}(K-V) \exp \left[\left(1+\frac{1}{2}\left(\hat{k}_{p}(K+V)+\hat{k}_{p}(K-V)\right)\right)^{p}\right] .
$$

Note that

$$
((K-V) f)(t)=\int_{x}^{1} k(t, s) f(s) d s .
$$

Corollary 5.1 implies

Corollary 5.2. Let $K$ be $a\left(p, p^{\prime}\right)$-Hille-Tamarkin integral operator in $L^{p}(0,1)$ for an integer $p \geq 2$ and $1 / p+$ $1 / p^{\prime}=1$. If $\hat{\Delta}_{p}(K, V)<1$, then

$$
\left|\operatorname{det}\left(I-K^{p}\right)-1\right| \leq \hat{\Delta}_{p}(K, V)
$$

and therefore $\sqrt[p]{1} \notin \sigma_{\Phi}(K)$, provided $\hat{\Delta}_{p}(K, V)<1$. 


\section{Hille-Tamarkin infinite matrices "close" to triangular ones}

Let us consider the linear operator $T$ in $l^{p}(2 \leq p<\infty)$ generated by an infinite matrix $\left(t_{j k}\right)_{j, k=1}^{\infty}$, satisfying the condition

$$
\tau_{p}(T):=\left[\sum_{j=1}^{\infty}\left(\sum_{k=1}^{\infty}\left|t_{j k}\right|^{p^{\prime}}\right)^{p / p^{\prime}}\right]^{1 / p}<\infty
$$

where $1 / p+1 / p^{\prime}=1$.

Then $T$ is called a $\left(p, p^{\prime}\right)$-Hille-Tamarkin matrix. As is well known, any $\left(p, p^{\prime}\right)$-Hille-Tamarkin matrix $T$ is an absolutely $p$-summing operator with $\pi_{p}(T) \leq \tau_{p}(T)$, cf. [8, p. 43], [2, Sections 5.3.2 and 5.3.3, p. 230] ). So according to (3.1),

$$
\sum_{k=1}^{\infty}\left|\lambda_{k}(T)\right|^{p} \leq \tau_{p}^{p}(T) \quad(2 \leq p<\infty)
$$

Let $T_{+}=\left(\tau_{j k}\right)_{j, k=1}^{\infty}$ be the upper-triangular part of $T: \tau_{j k}=t_{j k}$ for $1 \leq j \leq k \leq \infty$ and $\tau_{j k}=0$ otherwise. Since $p>p^{\prime}$, we obtain

$$
\left(\sum_{k=1}^{\infty}\left|t_{j k}\right|^{p^{\prime}}\right)^{p / p^{\prime}} \geq \sum_{k=1}^{\infty}\left|t_{j k}\right|^{p}
$$

and thus

$$
\sum_{j=1}^{\infty} \sum_{k=1}^{\infty}\left|t_{j k}\right|^{p}<\infty .
$$

Since $T_{+}$is triangular, its eigenvalues are the diagonal entries and

$$
\operatorname{det}\left(I-T_{+}^{p}\right)=d_{+, p}:=\prod_{k=1}^{\infty}\left(1-t_{k k}^{p}\right) .
$$

Under consideration,

$$
\Delta_{p}\left(T, T_{+}\right)=\pi_{p}\left(T-T_{+}\right) \exp \left[\left(1+\frac{1}{2}\left(\pi_{p}\left(T+T_{+}\right)+\pi_{p}\left(T-T_{+}\right)\right)\right)^{p}\right] \leq \hat{\Delta}_{p}\left(T, T_{+}\right),
$$

where

$$
\hat{\Delta}_{p}\left(T, T_{+}\right):=\tau_{p}\left(T-T_{+}\right) \exp \left[\left(1+\frac{1}{2}\left(\tau_{p}\left(T+T_{+}\right)+\tau_{p}\left(T-T_{+}\right)\right)\right)^{p}\right] .
$$

Note that $T-T_{+}$is the strictly lower part of $T$.

Making use of Lemma 2.1, we arrive at

Corollary 6.1. Let $T$ be $a\left(p, p^{\prime}\right)$-Hille-Tamarkin matrix for an integer $p \geq 2$ and $1 / p+1 / p^{\prime}=1$. Then $\mid \operatorname{det}(I-$ $\left.T^{p}\right)-d_{+, p} \mid \leq \hat{\Delta}_{p}\left(T, T_{+}\right)$, and therefore $\sqrt[p]{1} \notin \sigma_{\Phi}(T)$, provided $\left|d_{+, p}\right|>\hat{\Delta}_{p}\left(T, T_{+}\right)$.

\section{References}

[1] Kato T., Perturbation Theory for Linear Operators, Springer-Verlag, 1980.

[2] Pietsch A., Eigenvalues and s-Numbers, Cambridge Univesity Press, 1987.

[3] König H., Eigenvalue Distribution of Compact Operators, Operator Theory: Advances and Applications, Basel: Birkhäuser, 1986.

[4] Gohberg I.C., Goldberg S., Krupnik N., Traces and Determinants of Linear Operators, Basel: Birkhäuser Verlag, 2000.

[5] Ciecierska G., Analytic formulae for determinant systems for a certain class of Fredholm operators in Banach spaces, Demonstratio Math., 1997, 30(2), 387-402.

[6] Gil' M.I., Bounds for Determinants of Linear Operators and Their Applications, CRC Press, Taylor \& Francis Group, 2017.

[7] Gil’ M.I., Perturbations of regularized determinants of operators in a Banach space, J. Math., 2013, Article ID 409604.

[8] Diestel J., Jarchow H., Tonge A., Absolutely Summing Operators, Cambridge University Press, 1995.

[9] Garling D.J., Inequalities: A Journey into Linear Analysis, Cambridge University Press, 2007. 
[10] Caetano A.M., Weyl numbers in function spaces, Forum Math., 1990, 2, 249-263.

[11] Carl B., On s-numbers, quasi s-numbers, s-moduli and Weyl inequalities of operators in Banach spaces, Rev. Mat. Comput, 2010, 23, 467-487.

[12] Carl B., Hinrichs A., Rudolph P., Entropy numbers of convex hulls in Banach spaces and applications, J. Complexity, 2014, 30(5), 555-587.

[13] Faried N., Abd El Ghaffar H., Approximation numbers of an infinite matrix operator from any Banach space with Schauder basis into itself, Int. J. Contemp. Math. Sci., 2012, 7(37), 1831-1838.

[14] Hinrichs A., Optimal Weyl inequalities in Banach spaces, In: Proc. Amer. Math. Soc., 2005, 134, 731-735.

[15] Rogozhin A., Silbermann B., On the approximation numbers for the finite sections of block Töeplitz matrices, Bull. London Math. Soc., 2006, 38(2), 301-313.

[16] Nguyen V.K., Sickel W., Weyl numbers of embeddings of tensor product Besov spaces, J. Approx. Theory, 2015, 200, 170220. 